\title{
Prolonged Exposure for PTSD in a Veterans Health Administration PTSD Clinic
}

\author{
Sheila A. M. Rauch \\ Mental Health Service, Veterans Administration Ann Arbor Healthcare System and Department \\ of Psychiatry, University of Michigan Medical School, Ann Arbor, MI \\ Erin Defever, Todd Favorite, Anne Duroe, and Cecily Garrity \\ Mental Health Service, Veterans Administration Ann Arbor Healthcare System, Ann Arbor, MI \\ Brian Martis and Israel Liberzon \\ Mental Health Service, Veterans Administration Ann Arbor Healthcare System and Department \\ of Psychiatry, University of Michigan Medical School, Ann Arbor, MI
}

\begin{abstract}
With the move toward dissemination of empirically supported treatments in the Veterans Health Administration (VHA), dissemination of additional data concerning the effectiveness of prolonged exposure (PE) among veterans is important. The authors present clinical treatment data from veterans with chronic posttraumatic stress disorder (PTSD) treated in a VHA PTSD clinic $(N=10)$. Veterans demonstrated significant reductions in total PTSD symptoms from pre-to posttreatment. Returning veterans from the conflicts in Afghanistan and Iraq and other era veterans (Vietnam Veterans and military sexual trauma veterans) demonstrated significant reductions in PTSD. In addition, veterans demonstrated significant reductions in depression from pre- to posttreatment. In conclusion, $P E$ is effective in reducing the symptoms of PTSD in veterans.
\end{abstract}

Prolonged exposure (PE), has proven effectiveness in the treatment of posttraumatic stress disorder (PTSD) symptoms associated with a variety of traumas (Rothbaum et al., 2000), including combat (Boudewyns \& Hyer, 1990; Glynn et al., 1999; Keane, Fairbank, Caddell, \& Zimering, 1989). Prolonged exposure significantly reduces PTSD symptoms, general anxiety, depression, guilt, and anger (Cahill, Rauch, Hembree, \& Foa, 2003; Foa et al., 2005). Prolonged exposure is a first line treatment for PTSD symptoms (Rothbaum et al., 2000; IOM, 2007) including a guideline for treatment of PTSD in returning veterans from Iraq and Afghanistan (Friedman, 2006).

Nevertheless, PE is often not accessible to veterans seeking PTSD treatment in the VA system. In a study examining treatment strategies in a region of the VA healthcare system, less than $10 \%$ of PTSD therapists routinely used PE (Rosen et al., 2004). They suggested a lack of therapists capable of providing PE, as well as misconceptions about PE may drive the deficit. The simplicity of PE including just three main therapeutic components (i.e., psychoeducation, in vivo exposure, and imaginal exposure) and the research supporting its efficacy make it an excellent candidate for dissemination and modification for use in varied settings (i.e., primary care, with comorbid conditions, etc.). In recognition of the importance of dissemination, the United States Veterans Health Administration (VHA) is conducting a roll-out of PE. Yet, dissemination of additional data concerning the effectiveness of $\mathrm{PE}$ among veterans is needed.

The current study presents clinical treatment data on a case series $(N=10)$ of veterans with chronic PTSD treated with PE in a PTSD Clinic in the VHA system. The clinic hired a clinician with expertise in $\mathrm{PE}$ and has been training providers and implementing this treatment option for veterans. All clinical cases treated with PE between January 2006 and December 2007 with available pre- and posttreatment PTSD severity scores are presented. We hypothesize that PTSD and depressive symptoms will be reduced from pre- to posttreatment.

\section{METHOD}

\section{Participants and Procedure}

Ten veterans (eight men, two women) diagnosed with chronic PTSD treated with PE in the PTSD Clinic (PCT) at the VA

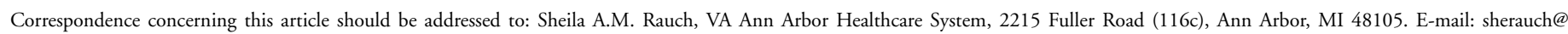
med.umich.edu. 
Table 1. Means on Symptom Measures

\begin{tabular}{lrrr}
\hline Variable & $M$ & \multicolumn{1}{c}{$S D$} & $n$ \\
\hline Dissociative Experiences Survey & 22.0 & 10.4 & 9 \\
State Trait Anger Inventory- Trait Scale & 25.3 & 5.7 & 9 \\
Posttraumatic Cognitions Inventory & 150.6 & 23.9 & 5 \\
\hline
\end{tabular}

Ann Arbor Healthcare System (VAAAHS) were included. The 10 veterans averaged 39 years of age $(S D=15)$. Five were married, two were remarried, and three were divorced or separated. Nine of the 10 were high school graduates (of whom three had some college), and six were employed. The average service connected disability rating was roughly $30 \%$. A majority $(70 \%)$ had at least one other Axis I diagnosis. Sixty percent were prescribed at least one psychoactive medication and $40 \%$ were taking three or more psychoactive medications.

All veterans had a comprehensive evaluation, including the Posttraumatic Diagnostic Scale (PDS; Foa, Cashman, Jaycox, \& Perry, 1997), BDI-II scores (Beck, Steer, \& Brown, 1996), Posttraumatic Cognitions Inventory (Foa, Tolin, Ehlers, Clark, \& Orsillo, 1999), Dissociative Experiences Scale (Carlson \& Putnam, 1993), Spielberger Trait Anger Inventory (Spielberger, 1999), Clinician Administered PTSD Scale (CAPS; Blake et al., 1995) and Mini International Neuropsychiatric Interview (MINI; Sheehan et al., 1998). The PDS has demonstrated high internal consistency $(\alpha=.92$ total $)$ and good test-retest reliability $(r=.74$ to .85$)$. The PDS contains 17 items and ranges from 0 to 51. A clinical cutoff score of 15 is used such that scores above 15 are likely to meet diagnosis of PTSD. All veterans met criteria for PTSD based on CAPS and PDS score at pretreatment (lowest PDS $=25$ ). Means on these measures are shown in Table 1.

Because the current analyses are from a clinical sample, formal inclusion and exclusion criteria were not utilized. Treatment plans and case assignments were made during clinic team meetings. Prolonged exposure was considered in the treatment plan for any veteran who met criteria for PTSD, was interested in receiving psychotherapy for PTSD, had at least one specific trauma memory, and did not have other clinical issues that required attention first (i.e., imminent suicidal risk, substance dependence, psychotic features, etc.). Cases that were identified as more difficult (i.e., involved significant substance use, personality disorders, recent dissociative tendencies, etc.) were assigned to the expert therapist (SR). Data presented represent all veterans with pre- and posttreatment PDS scores. Pre- and posttreatment BDI-II scores are included when available. Although additional veterans have been treated, data was not available for either pre- or posttreatment PDS. In addition, rates of dropout and data on veterans who dropped out are not available. All veterans with available data were Caucasian.
According to the MINI, $80 \%$ of the sample had at least one other Axis I diagnosis according to the Diagnostic and Statistical Manual of Mental Disorders, Fourth Edition (DSM-IV; American Psychiatric Association, 1994), including current major depressive disorder (50\%), bipolar disorder (20\%), alcohol/drug dependence $(20 \%)$, social anxiety disorder $(10 \%)$, and $10 \%$ with another Axis 1 disorder. Medication was observed and recorded, but was managed irrespective of PE. At the time of PE initiation, $70 \%$ of veterans were prescribed at least one psychoactive medication. Fifty percent were prescribed at least three psychoactive medications and $20 \%$ were prescribed four or more psychoactive medications. Of those veterans on medication over the course of $\mathrm{PE}$, three veterans had medication increases, one veteran discontinued medication, and three veterans had no change to medication.

The first author trained all of the clinical staff. Half of the cases $(n=5)$ served as first training cases for therapists with no previous experience with PE and minimal cognitive-behavioral therapy (CBT) training. The trainees included a psychology postdoctoral fellow (just received doctorate), an experienced Masters-level social worker (7 years in clinical practice), a Masters-level social worker who obtained her license during training, and two psychology advanced practicum students (currently working toward their doctorates). The PE training program involved four 2-hour didactic sessions covering the basic research, rationale, primary components, and role plays. The standard of training used was that each trainee was required to complete two training cases. For each case, the sessions were videotaped and viewed by the supervisor who provided written feedback and met with the therapist for 30 minutes per session. The current data include first training cases as well as cases seen by the first author.

A modification to the standard PE manual (Foa et al., 2007) written by the first author and Dr. Edna Foa was used. Sessions were reduced to 80 minutes and wording was changed to reflect the population. All veterans received between 7 and 21 sessions $(M=12.7 ; S D=4.5)$. Variability in number of sessions is due to the therapist and/or patient deciding to end therapy due to symptom reduction (eight cases) or other factors (i.e., starting a new job and unable to attend sessions; two cases).

\section{RESULTS}

To examine changes in PTSD symptoms among the 10 veterans, 4 (total PDS, reexperiencing, avoidance numbing, and hyperarousal) separate dependent $t$ tests comparing pre- and post-PDS scores were conducted (see Table 2). Effect size was then calculated using Cohen's $d$ (Cohen, 1969). Veterans demonstrated significant reductions in total PTSD symptoms from pre- $(M=36.2, S D=$ 8.1) to posttreatment $(M=17.2, S D=9.2), t(9)=4.69, p<.01$, Cohen's $d=2.19$. Cohen's $d$ was large (Cohen, 1969). Fifty percent of treated veterans had PDS scores below 15 suggesting they no longer met criteria for PTSD. 
Table 2. Pre- and Posttreatment Posttraumatic Stress Disorder and Depression Severity

\begin{tabular}{|c|c|c|c|c|c|c|c|c|c|c|c|c|}
\hline \multirow[b]{2}{*}{ Target trauma } & \multicolumn{3}{|c|}{ Pre-PDS } & \multicolumn{3}{|c|}{ Post-PDS } & \multicolumn{3}{|c|}{ Pre-BDI-II } & \multicolumn{3}{|c|}{ Post-BDI-II } \\
\hline & $M$ & $S D$ & $n$ & $M$ & $S D$ & $n$ & $M$ & $S D$ & $n$ & M & $S D$ & $n$ \\
\hline Vietnam & 38.3 & 12.6 & 3 & 18.0 & 16.1 & 3 & 19.6 & 9.1 & 3 & 3.5 & 0.7 & 2 \\
\hline OEF/OIF & 34.2 & 7.5 & 5 & 16.0 & 7.5 & 5 & 22.0 & 4.6 & 3 & 15.0 & 4.4 & 3 \\
\hline MST & 38.0 & 2.8 & 2 & 19.0 & 1.4 & 2 & 36.0 & 11.3 & 2 & 22.0 & 5.7 & 2 \\
\hline Vietnam/MST & 38.2 & 9.0 & 5 & 18.4 & 11.4 & 5 & 26.2 & 12.4 & 5 & 12.8 & 11.2 & 4 \\
\hline Total & 36.2 & 8.1 & 10 & 17.2 & 9.2 & 10 & 24.6 & 9.9 & 8 & 13.0 & 8.4 & 7 \\
\hline
\end{tabular}

Note. PDS = Posttraumatic Diagnostic Scale; BDI-II = Beck Depression Inventory-2nd ed.; OEF = Operation Enduring Freedom; OIF = Operation Iraqi Freedom; $\mathrm{MST}=$ Military Sexual Trauma.

To examine reliable and clinically significant change, a reliable change score criterion and clinical significance criterion were calculated for the PDS (Jacobson \& Truax, 1991). Using the normative data for the PDS (Foa et al., 1997), change that exceeded 7.8 points is considered reliable change. In this sample, $90 \%$ of veterans demonstrated reliable reduction in PTSD from pre- to posttreatment. Using the clinical significance criterion of moving to the normal side of the point halfway between PTSD and non-PTSD normative samples, posttreatment scores below 23.4 are clinically significant. In this sample, $80 \%$ of veterans demonstrated clinically significant reduction in PTSD.

With regard to subscales, veterans demonstrated significant reductions in reexperiencing symptoms (pretreatment: $M=10.9$, $S D=3.2$; posttreatment: $M=4.9, S D=4.2), t(7)=3.42$, $p<.01, d=1.60$; avoidance symptoms (pretreatment: $M=13.5$, $S D=4.3$, to posttreatment: $M=5.9, S D=4.3), t(7)=3.50$, $p<.05, d=1.77$, and hyperarousal symptoms (pretreatment: $M=12.4, S D=1.9$; posttreatment: $M=6.0, S D=3.51$ ), $t(7)=4.12, p<.01, d=2.28$.

The small sample size in this study does not allow for comparison of returning veterans from Afghanistan and Iraq (RV) to other era veterans (Vietnam veterans [VV] and military sexual trauma $[\mathrm{MST}]$ veterans). Both groups demonstrated significant reductions from pretreatment to posttreatment, $\mathrm{RV}, t(4)=3.60, p<.05$, $d=2.42$; VV/MST, $t(4)=2.90, p<.05, d=1.93$. Of note, $60 \%$ of RVs and $40 \%$ of the VV/MST had PDS scores below 15 suggesting they no longer met criteria for PTSD. Responses of the veterans are depicted in Figure 1.

In addition to PTSD, 7 of 10 veterans had pre- and posttreatment BDI-II scores. To examine changes in depressive symptoms, a dependent $t$ test comparing pre- and post-BDI-II scores was conducted. Veterans demonstrated significant reductions in total depressive symptoms from pre- $(M=25.9, S D=10.0)$ to posttreatment $(M=13.7, S D=8.4), t(6)=4.07, p<.05, d=1.31$. Cohen's $d$ was large (Cohen, 1969).

To examine reliable and clinically significant change, a reliable change score criterion and clinical significance criterion were calculated for the BDI-II (Jacobson \& Truax, 1991). Using the normative data for the BDI-II (Beck et al., 1996), change that exceeded 9.20 points is considered reliable change. In this sample, $40 \%$ of veterans demonstrated reliable reduction in depression from pre- to posttreatment. Using the clinical significance criterion of moving to the normal side of the point halfway between the depressed and nondepressed normative samples, posttreatment scores below 14.9 are clinically significant. In this sample, $40 \%$ of veterans demonstrated clinically significant reduction in depression from pre- to posttreatment.

Finally, to address whether trainee cases differed in outcome from the cases of the experienced PE clinician, two independent $t$ tests were conducted with clinician status (trainee vs. experienced) as the independent variable and PDS and BDI-II scores as the dependent variables. No differences were detected, PDS, $t(8)=$ $1.80, n s$; BDI-II, $t<1$.

\section{DISCUSSION}

Prolonged exposure is effective in reducing the symptoms of PTSD in veterans receiving care in a VHA PTSD clinic. Reductions in symptoms were large across all three symptom clusters. Effect sizes (overall $d=2.19$ ) are consistent with those found in randomized clinical trials with nonveteran and veteran populations. Specifically, the pre- to posttreatment within-group effect sizes found for $\mathrm{PE}$ in trials range from a low of .8 in a study of female veterans (Schnurr et al., 2007) to a high of 2.3 in a study of sexual assault survivors (Resick, Nishith, Weaver, Astin, \& Feuer, 2002).

Several additional points are to be noted. First, VV/MST and RVs responded well to PE. Second, trainees with limited CBT experience who received 8 hours of didactic training in PE treated $50 \%$ of the sample with excellent results. Severe comorbidities were ruled out as training cases; however, the sample still had significant comorbidity ( $80 \%$ met criteria for at least one other diagnosis). Although power may be an issue due to the small sample size, no differences in reduction of PTSD or depressive symptoms were detected between the experienced PE clinician and the trainees. These preliminary findings with recently trained therapists are 


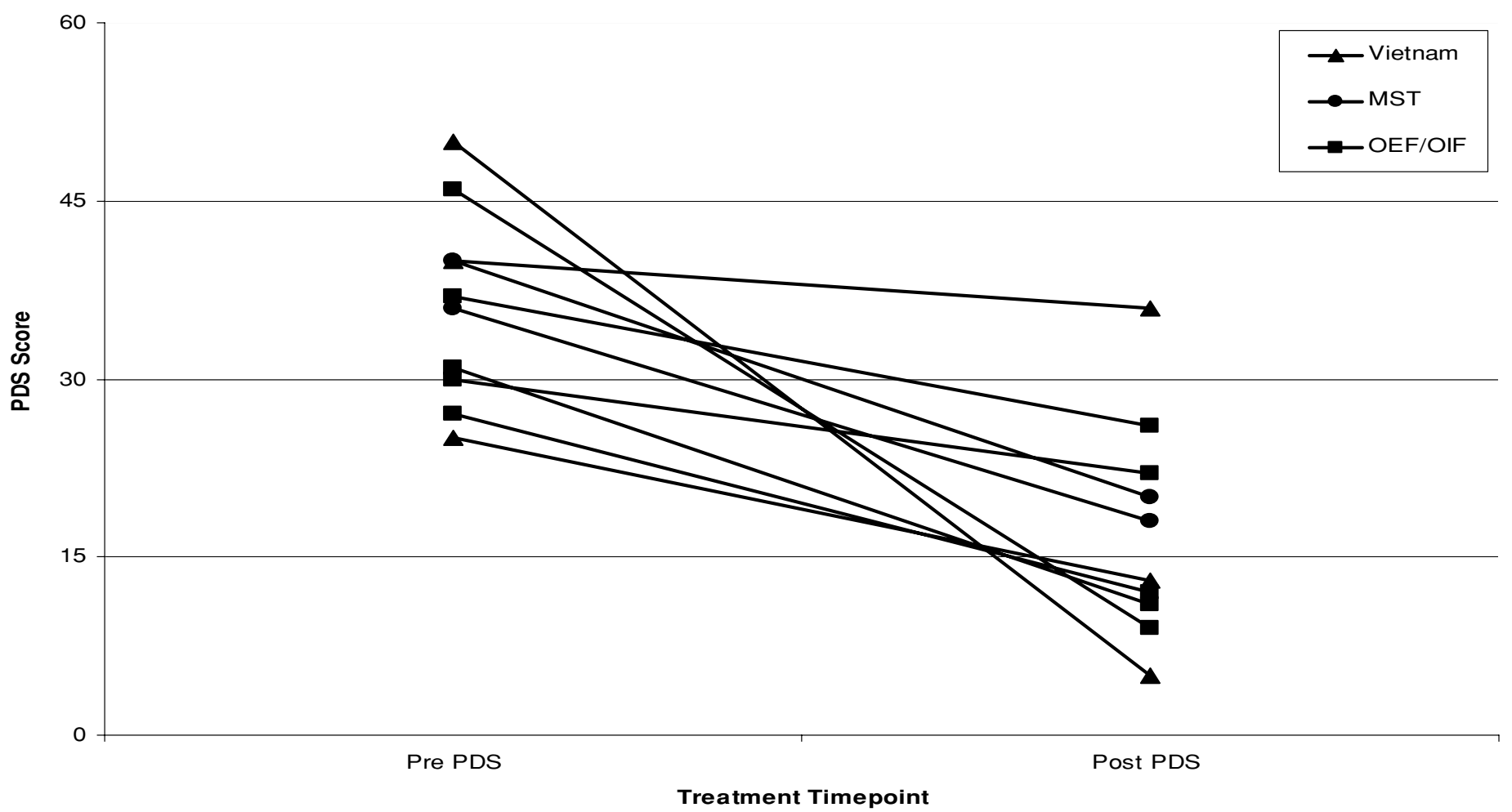

Figure 1. Veteran treatment change by trauma type. PDS = Posttraumatic Diagnostic Scale; OEF = Operation Enduring Freedom; OIF $=$ Operation Iraqi Freedom; MST = Military Sexual Trauma.

encouraging in the context of training a larger number of therapists nationwide.

Several caveats to the interpretation of these findings are warranted. First, the sample size is small. Second, these results are based on self-report data that may be subject to response bias. Third, these are clinical data collected in a VA PTSD clinic. As such, many factors were not controlled (i.e., medication, comorbidity, etc.), randomization was not possible, and attrition rates cannot be obtained. Fourth, adherence data was not collected on these cases. Although five of the cases were training cases that were viewed by the first author and detailed feedback provided to the therapist on violations of standard protocol, we do not know how precisely the therapists complied with the manual. In clinical practice as PE is rolled out, it is unlikely that trainee therapists will have adherence data beyond supervisor feedback. As such, these cases represent a real-time clinical application of PE. Finally, all of the veterans in this sample were Caucasian. This lack of racial/ethnic diversity is consistent with the clinic demographics (about $83 \%$ Caucasian), but we do not know whether these results will apply to other racial/ethnic groups.

These data support the efficacy of PE for use in VA PTSD clinics; nevertheless, many barriers remain. One significant barrier that is currently being surmounted is the limited availability of training. The VHA PE Roll Out initiative promises to provide training to hundreds of clinicians and create a national network of PE trainers and supervisors.

Clinicians often harbor concerns about using PE with many types of veterans or feel unsure about how to make a decision about whether to use PE or other alternative treatments (e.g., cognitive processing therapy, stress inoculation training, supportive contacts, or crisis management). For instance, in the work of the first author training clinicians in PE, many trainees believe that $\mathrm{PE}$ is not effective for survivors of multiple incident trauma, such as combat. However, several studies have supported the efficacy of PE for PTSD resulting from multiple incident trauma (Glynn et al., 1999; McDonagh et al., 2005; Schnurr et al., 2007). Another concern voiced is that PE is less tolerable than other PTSD treatments. However, a meta-analysis of treatment dropout suggests that rates of dropout are similar for PE $(20.5 \%)$ and other CBT treatments (22-27\%) and medications (22-38\%; Connor, Sutherland, Tupler, Malik, \& Davidson, 1999; Hembree et al., 2003; Tucker et al., 2001). Although research can provide some direction, additional studies are needed to examine how and why PE and other PTSD treatments work to improve treatment and assist clinicians in making informed decisions about treatment options. 
Logistics of the VA clinic structure may impede the implementation of PE. For instance, clinician case-load expectations often do not allow for time to train in a new therapy. Further, supervision from an expert requires both trainee and supervisor time. Most VA clinics function based on a standard 60-minute clinical hour with the number of unique veterans contacted used as the metric for case load. This does not allow for longer and more frequent contacts with just a single veteran. Although these logistic issues require attention, recent VA Office of Mental Health policy changes have placed a premium on the provision of PE (as well as other treatments) to our veterans and promise to provide the resources needed to implement PE and other empirically supported treatments. This roll-out promises to result in improved treatment outcomes and potentially more efficient treatment for veterans with PTSD.

\section{REFERENCES}

American Psychiatric Association. (1994). Diagnostic and statistical manual of mental disorders (4th ed.). Washington, DC: Author.

Beck, A. T., Steer, R. A., \& Brown, G. K. (1996). Beck Depression Inventory Manual (2nd ed.). San Antonio, TX: The Psychological Corporation.

Blake, D. D., Weathers, F. W., Nagy, L. M., \& Kaloupek, D. G. (1995). The development of a Clinician-Administered PTSD Scale. Journal of Traumatic Stress, 8(1), 75-90.

Boudewyns, P. A., \& Hyer, L. (1990). Physiological response to combat memories and preliminary treatment outcome in Vietnam veteran PTSD patients treated with direct therapeutic exposure. Behavior Therapy, 21, 6387.

Cahill, S. P., Rauch, S. A., Hembree, E. A., \& Foa, E. B. (2003). Effect of cognitivebehavioral treatments for PTSD on anger. Journal of Cognitive Psychotherapy, $17,113-131$.

Carlson, E. B., \& Putnam, F. W. (1993). An update on the Dissociative Experiences Scale. Dissociation, 6, 16-27.

Cohen, J. (1969). Statistical power analysis for behavioral sciences. New York: Academy Press.

Connor, K. M., Sutherland, S. M., Tupler, L. A., Malik, M. L., \& Davidson, J. R. T. (1999). Fluoxetine in post-traumatic stress disorder: Randomised, double-blind study. British Journal of Psychiatry, 175, 17-22.

Foa, E. B., Cashman, L., Jaycox, L., \& Perry, K. (1997). The validation of a selfreport measure of posttraumatic stress disorder: The Posttraumatic Diagnostic Scale. Psychological Assessment, 9, 445-451.

Foa, E. B., Hembree, E. A., Cahill, S. P., Rauch, S. A. M., Riggs, D. S., Feeny, N. C., et al. (2005). Randomized trial of prolonged exposure for posttraumatic stress disorder with and without cognitive restructuring: Outcome at academic and community clinics. Journal of Consulting and Clinical Psychology, 73, 953-964.

Foa, E. B., Tolin, D. F., Ehlers, A., Clark, D. M., \& Orsillo, S. M. (1999). The Posttraumatic Cognitions Inventory (PTCI): Development and validation. Psychological Assessment, 11, 303-314.

Friedman, M. J. (2006). Posttraumatic stress disorder among military returnees from Afghanistan and Iraq. American Journal of Psychiatry, 163, 586-593.

Glynn, S. M., Eth, S., Randolph, E. T., Foy, D. W., Urbaitis, M., Boxer, L., et al. (1999). A test of behavioral family therapy to augment exposure for combat-related posttraumatic stress disorder. Journal of Consulting and Clinical Psychology, 67, 243-251.

Hembree, E. A., Foa, E. B., Dorfan, N. M., Street, G. P., Kowalski, J., \& Tu, X. (2003). Do patients drop out prematurely from exposure therapy for PTSD? Journal of Traumatic Stress, 16, 555-562.

Institute of Medicine. (2007). Treatment of PTSD: An assessment of the evidence. Washington, DC: Author.

Jacobson, N. S., \& Truax, P. (1991). Clinical significance: A statistical approach to defining meaningful change in psychotherapy research. Journal of Consulting and Clinical Psychology, 59, 12-19.

Keane, T. M., Fairbank, J. A., Caddell, J. M., \& Zimering, R. T. (1989). Implosive (flooding) therapy reduces symptoms of PTSD in Vietnam combat veterans. Behavior Therapy, 20, 245-260.

McDonagh, A., Friedman, M., McHugo, G., Ford, J., Sengupta, A., Mueser, K., et al. (2005). Randomized trial of cognitive-behavioral therapy for chronic posttraumatic stress disorder in adult female survivors of childhood sexual abuse. Journal of Consulting and Clinical Psychology, 73, 515-524.

Resick, P. A., Nishith, P., Weaver, T. L., Astin, M. C., \& Feuer, C. A. (2002). A comparison of cognitive-processing therapy with prolonged exposure and a waiting list condition for the treatment of chronic PTSD in female rape victims. Journal of Consulting and Clinical Psychology, 70, 867-879.

Rosen, C. S., Chow, H. C., Finney, J. F., Greenbaum, M. A., Moos, R. H., Sheikh, J. I., et al. (2004). VA practice patterns and practice guidelines for treating posttraumatic stress disorder. Journal of Traumatic Stress, 17, 213-222.

Rothbaum, B. O., Meadows, E. A., Resick, P., Foy, D. W., Foa, E. B., Keane, T. M., et al. (2000). Cognitive-behavioral therapy. New York: Guilford Press.

Schnurr, P. P., Friedman, M. J., Engel, C. C., Foa, E. B., Shea, M. T., Chow, B. K., et al. (2007). Cognitive behavioral therapy for posttraumatic stress disorder in women: A randomized controlled trial. Journal of the American Medical Association, 297, 820-830.

Sheehan, D. V., Lecrubier, Y., Sheehan, K. H., Amorim, P., Janavs, J., Weiller, E., et al. (1998). The Mini-International Neuropsychiatric Interview (M.I.N.I): The development and validation of a structured diagnostic psychiatric interview for DSM-IV and ICD-10. Journal of Clinical Psychiatry, 59(20), 22-33.

Spielberger, C. D. (1999). STAXI-2-State Trait Anger Expression Inventory-2: Professional Manual. Lutz, FL: Psychological Assessment Resources, Inc.

Tucker, P., Zaninelli, R., Yehuda, R., Ruggiero, L., Dillingham, K., \& Pitts, C. D. (2001). Paroxetine in the treatment of chronic posttraumatic stress disorder: Results of a placebo-controlled, flexible-dosage trial. Journal of Clinical Psychiatry, 62, 860-868. 